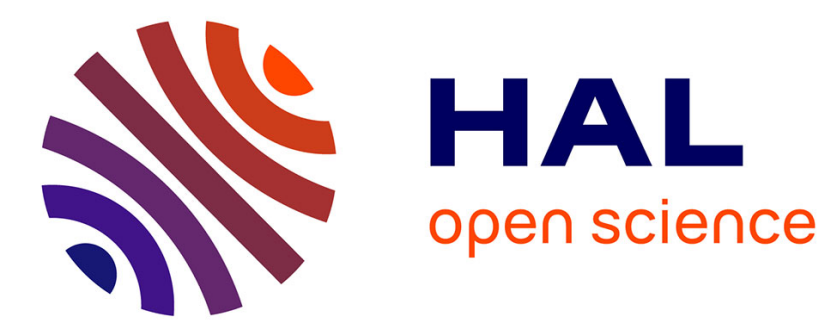

\title{
On products of ratios of consecutive integers
}

Régis de La Bretèche, Carl Pomerance, Gérald Tenenbaum

\section{To cite this version:}

Régis de La Bretèche, Carl Pomerance, Gérald Tenenbaum. On products of ratios of consecutive integers. Ramanujan Journal (The), 2005, 9, pp.131-138. hal-00091182

\section{HAL Id: hal-00091182 \\ https://hal.science/hal-00091182}

Submitted on 5 Sep 2006

HAL is a multi-disciplinary open access archive for the deposit and dissemination of scientific research documents, whether they are published or not. The documents may come from teaching and research institutions in France or abroad, or from public or private research centers.
L'archive ouverte pluridisciplinaire HAL, est destinée au dépôt et à la diffusion de documents scientifiques de niveau recherche, publiés ou non, émanant des établissements d'enseignement et de recherche français ou étrangers, des laboratoires publics ou privés. 


\title{
Products of ratios of consecutive integers
}

\author{
Régis de la Bretèche, Carl Pomerance \& Gérald Tenenbaum
}

For Jean-Louis Nicolas, on his sixtieth birthday

\section{Introduction}

Let $\left\{\varepsilon_{n}\right\}_{1 \leqslant n<N}$ be a finite sequence with each $\varepsilon_{n} \in\{0, \pm 1\}$, and write

$$
\frac{a}{b}=\prod_{1 \leqslant n<N}\left(\frac{n}{n+1}\right)^{\varepsilon_{n}},
$$

where the fraction is in its smallest terms. Now, define $A(N)$ as the maximal value of $a$ as $\left\{\varepsilon_{n}\right\}_{1 \leqslant n<N}$ runs through all possible $3^{N-1}$ sequences of $0, \pm 1$, and let $B(N)$ denote the corresponding value of $b$. (Note that maximizing $b$ instead of $a$ would lead to $B(N) / A(N)$.) We obviously have $A(N) \leqslant N$ !, hence $\log A(N) \leqslant N \log N$ for all $N$. In [7], it is shown by an elegant "near-tiling" of the integers in $[1, N]$ with triples $n, 2 n, 2 n+1$ that

$$
\log A(N) \leqslant\left\{\frac{2}{3}+o(1)\right\} N \log N .
$$

Further, a brief argument of M. Langevin is presented that

$$
\log A(N) \geqslant\{\log 4+o(1)\} N .
$$

Our aim in this article is to establish the true order of magnitude for $\log A(N)$. Put

$$
\begin{aligned}
k(c) & :=1+2 \log (1-2 c)-\frac{2}{c} \log \left(1+\frac{2 c^{2}}{1-3 c}\right), \\
K(c) & :=2 \int_{0}^{c} k(u) \mathrm{d} u, \quad K:=\max _{0<c<1 / 5} K(c) \approx 0.107005 .
\end{aligned}
$$

Theorem 1.1. For large $N$, we have

$$
\log A(N) \geqslant\{K+o(1)\} N \log N
$$

Let $P(n)$ denote the largest prime factor of a positive integer $n$ with the convention that $P(1)=1$. The lower bound $(1 \cdot 1)$ is an easy consequence of the estimate stated in the following result.

Theorem 1.2. For $c \in[0,1], x \geqslant 1$, let $S(x, c)$ denote the number of those integers $n$ not exceeding $x$ such that $\min \{P(n), P(n+1)\}>x^{1-c}$. Then, for any fixed $\left.c_{0} \in\right] 0, \frac{1}{5}\left[\right.$ and uniformly for $c \in\left[0, c_{0}\right], x \rightarrow \infty$, we have

$$
S(x, c) \leqslant 2 x \int_{0}^{c} \log \left(\frac{1-v}{1-v-2 c}\right) \frac{\mathrm{d} v}{1-v}+o(x) .
$$


Remark. Under a suitably strong form of the Elliott-Halberstam hypothesis, we get the better bound

$$
S(x, c) \leqslant x\{\log (1-c)\}^{2}+o(x)
$$

for $0 \leqslant c \leqslant \frac{1}{2}$. This would yield the value $K=4 / \sqrt{\mathrm{e}}-2 \approx 0.426123$ in Theorem 1.1. See Section 3 for further methodological remarks.

The bound (1.3) is probably optimal, in fact it is likely to be the case that $S(x, c)=x\{1-\varrho(1 /\{1-c\})\}^{2}+o(x)$ for $0 \leqslant c<1$, where $\varrho$ is the Dickman-de Bruijn function. An even stronger statement is suggested in [3].

Note that (1.1) follows from (1.2) by selecting $\varepsilon_{n}=1$ if $P(n)>N^{1-c}$ and $P(n)>P(n+1), \varepsilon_{n}=-1$ if $P(n+1)>N^{1-c}$ and $P(n+1)>P(n)$ and $\varepsilon_{n}=0$ in all other cases. Indeed, with these choices for $\varepsilon_{n}$, we obtain that for each prime $p>N^{1-c} \geqslant N^{1 / 2}$, the exponent on $p$ in the prime factorization of the rational number $A(N) / B(N)$ is

Thus,

$$
\sum_{\substack{n<N \\ P(n)=p}} 2-\sum_{\substack{n<N \\ P(n+1)>P(n)=p}} 2-\sum_{\substack{n<N \\ P(n)>P(n+1)=p}} 2 .
$$

$$
\log A(N) \geqslant \sum_{\substack{n<N \\ P(n)>N^{1-c}}} 2 \log P(n)-\sum_{\substack{n<N \\ P(n), P(n+1)>N^{1-c}}} 2 \log \min \{P(n), P(n+1)\} .
$$

We have

$$
\begin{array}{r}
\sum_{\substack{n \leqslant N \\
P(n), P(n+1)>N^{1-c}}} 2 \log \min \{P(n), P(n+1)\}=\int_{0}^{c}(1-u) \log N \mathrm{~d} S(N, u) \\
=(\log N)\left\{(1-c) S(N, c)+\int_{0}^{c} S(N, u) \mathrm{d} u\right\},
\end{array}
$$

and, since the number of $n<N$ with $P(n)>N^{1-c}$ is $N \log \{1 /(1-c)\}+o(N)$ uniformly for $0 \leqslant c \leqslant 1 / 2$,

We thus obtain

$$
\sum_{\substack{n<N \\ P(n)>N^{1-c}}} \log P(n)=c N \log N+o(N) .
$$

$$
\begin{aligned}
\log A(N) & \geqslant 2(\log N)\left\{c N-(1-c) S(N, c)-\int_{0}^{c} S(N, u) \mathrm{d} u+o(N)\right\} \\
& \geqslant 2 N(\log N)\{g(c)+o(1)\},
\end{aligned}
$$

where we have set

$g(c):=c-(1-c) f(c)-\int_{0}^{c} f(u) \mathrm{d} u, \quad$ with $\quad f(u):=2 \int_{0}^{u} \log \left(\frac{1-v}{1-v-2 u}\right) \frac{\mathrm{d} v}{1-v}$.

We check by computation that $g^{\prime}(c)=k(c)$. This implies the desired estimate. 


\section{Proof of Theorem 1.2}

We employ the Rosser-Iwaniec sieve. A slightly better numerical bound could be obtained from a more sophisticated sieve method. We do not pursue such an improvement here but provide precise indications in Section 3. We refer to [5], [6] for a complete reference of the Rosser-Iwaniec coefficients and merely recall the property we shall use. We denote by $\gamma$ the Euler constant, and we let $p$ run over primes.

Lemma 2.1. Let $Q$ denote a set of primes, let $z \geqslant 2$ and write $Q(z):=\prod_{p \leqslant z, p \in Q} p$. There exists a sequence $\left\{\lambda_{d}\right\}_{d=1}^{\infty}$ of real numbers, vanishing for $d>z$ or $\mu(d)=0$, satisfying $\lambda_{1}=1,\left|\lambda_{d}\right| \leqslant 1, \mu * \mathbf{1} \leqslant \lambda * \mathbf{1}$, and such that for any number $\alpha>0$,

$$
\sum_{d \mid Q(z)} \frac{\lambda_{d} w(d)}{d} \leqslant \prod_{\substack{p \leqslant z \\ p \in \mathcal{Q}}}\left(1-\frac{w(p)}{p}\right)\left\{2 \mathrm{e}^{\gamma}+O_{\alpha}\left(\frac{1}{(\log z)^{1 / 3}}\right)\right\}
$$

uniformly for all multiplicative functions $w$ satisfying

$$
\text { (ii) } \prod_{u<p \leqslant v, p \in \mathcal{Q}}\left(1-\frac{w(p)}{p}\right)^{-1} \leqslant \frac{\log v}{\log u}\left(1+\frac{\alpha}{\log u}\right) \quad(2 \leqslant u \leqslant v \leqslant z) .
$$

If $n$ is counted by $S(x, c)$, then $n=a p_{1}=b p_{2}-1$, where $p_{1}$ and $p_{2}$ are primes greater than $x^{1-c}$. Then $a$ and $b$ are obviously coprime, and moreover $2 \mid a b$. We need an upper bound for the number $Z(a, b)$ of admissible pairs $\left(p_{1}, p_{2}\right)$ for given $a, b$. Let $C$ be a sufficiently large constant and set $z:=(x / a)^{1 / 2} b^{-1}(\log x)^{-C}$. If $\mathcal{Q}$ is the set of all primes not dividing $a$ and with $\left\{\lambda_{d}\right\}_{d=1}^{\infty}$ the sequence from Lemma 2.1, we plainly have

$$
\begin{aligned}
Z(a, b) & \leqslant \sum_{\substack{p_{1} \leqslant x / a \\
a p_{1} \equiv-1(\bmod b)}} \mu * \mathbf{1}\left(\left(a p_{1}+1\right) / b, Q(z)\right) \\
& \leqslant \sum_{d \mid Q(z)} \lambda_{d} \sum_{\substack{p_{1} \leqslant x / a \\
a p_{1} \equiv-1(\bmod b d)}} 1 .
\end{aligned}
$$

Let us put, for real $y \geqslant 2$ and integers $q, r$ with $q \geqslant 1$,

$$
\pi(y ; q, r):=\sum_{\substack{p \leqslant y \\ p \equiv r(\bmod q)}} 1, \quad E(y ; q):=\max _{(r, q)=1}|\pi(y ; q, r)-\operatorname{li}(y) / \varphi(q)| .
$$

We apply Lemma 2.1 to the multiplicative function $d \mapsto d \varphi(b) / \varphi(b d)$. Using the fact that $(a, b d)=1$ for each $d \mid Q(z)$, and noticing that $c$ bounded below $1 / 5$ ensures that $z \geqslant b$ when $x$ is large enough, we deduce that

$$
Z(a, b) \leqslant M(a, b)+R(a, b)
$$


with

$$
R(a, b):=\sum_{d \leqslant z} E(x / a ; b d)
$$

and

$$
\begin{aligned}
M(a, b) & :=\sum_{d \mid Q(z)} \frac{\lambda_{d} \operatorname{li}(x / a)}{\varphi(b d)} \\
& \leqslant\left\{2 \mathrm{e}^{\gamma}+o(1)\right\} \frac{\operatorname{li}(x / a)}{\varphi(b)} \prod_{\substack{p \leqslant z \\
p \nmid a b}}\left(1-\frac{1}{p-1}\right) \prod_{\substack{p \leqslant z \\
p \mid b}}\left(1-\frac{1}{p}\right) \\
& =\left\{2 \mathrm{e}^{\gamma}+o(1)\right\} \frac{\operatorname{li}(x / a)}{b} \prod_{\substack{p \leqslant z \\
p \nmid a b}}\left(\frac{p-2}{p-1}\right) .
\end{aligned}
$$

Now we observe that, uniformly as $x$ tends to $\infty$ and $a, b$ vary in the specified ranges,

$$
\prod_{\substack{p \leqslant z \\ p>2}}\left(\frac{p-2}{p-1}\right)=2 \prod_{\substack{p \leqslant z \\ p>2}} \frac{p(p-2)}{(p-1)^{2}} \prod_{p \leqslant z}\left(1-\frac{1}{p}\right) \sim \frac{2 \mathrm{e}^{-\gamma}}{A \log z}
$$

where

$$
A:=\prod_{p>2}\left(1+\frac{1}{p(p-2)}\right) .
$$

Therefore, writing

$$
h(n):=\prod_{\substack{p \mid n \\ p>2}}\left(\frac{p-1}{p-2}\right),
$$

we obtain that the estimate

$$
M(a, b) \leqslant \frac{\{8+o(1)\} h(a b) x}{A a b \log (x / a) \log \left(x / a b^{2}\right)}
$$

holds uniformly for $a \leqslant x^{c}, b \leqslant x^{c},(a, b)=1$, as $x \rightarrow \infty$.

Let $\tau(m)$ denote the number of divisors of $m$. By the Bombieri-Vinogradov theorem, we have, with $X_{a}:=(x / a)^{1 / 2}(\log x)^{-C}$,

$$
\begin{aligned}
\sum_{b \leqslant x^{c}} R(a, b) & \leqslant \sum_{m \leqslant X_{a}} \tau(m) E(x / a ; m) \\
& \ll\left\{\sum_{m \leqslant X_{a}} E(x / a ; m) \sum_{m \leqslant X_{a}} \tau(m)^{2} E(x / a ; m)\right\}^{1 / 2} \ll \frac{x}{a(\log x)^{2}},
\end{aligned}
$$


where we have used the trivial estimate $E(x / a ; m) \ll x / a m$ and the well-known fact that $\sum_{m \leqslant x} \tau(m)^{2} / m \ll(\log x)^{4}$. Therefore, we obtain from (2.2) and $(2 \cdot 3)$

$$
\begin{aligned}
S(x, c) & \leqslant \sum_{\substack{a \leqslant x^{c}, b \leqslant x^{c} \\
(a, b)=1,2 \mid a b}} Z(a, b) \\
& \leqslant \frac{8+o(1)}{A} x \sum_{a \leqslant x^{c}} \frac{h(a)}{a \log (x / a)} \sum_{\substack{b \leqslant x^{c} \\
2 \mid a b \\
(b, a)=1}} \frac{h(b)}{b \log \left(x / a b^{2}\right)}+O\left(\frac{x}{\log x}\right) .
\end{aligned}
$$

We have for $\nu=0$ or 1

$$
\sum_{\substack{b \geqslant 1 \\(b, a)=1}} \frac{h\left(2^{\nu} b\right)}{b^{s}}=H(s) G_{a}(s) \zeta(s) \quad(\Re e s>1)
$$

where

$$
H(s):=\prod_{p>2}\left(1+\frac{1}{p^{s}(p-2)}\right), \quad G_{a}(s):=\left(1-\frac{\varepsilon(a)}{2^{s}}\right) \prod_{\substack{p \mid a \\ p>2}}\left(\frac{1-p^{-s}}{1+p^{-s} /(p-2)}\right),
$$

with $\varepsilon(a)=1$ if $a$ is even, $\varepsilon(a)=0$ if $a$ is odd. The functions $H$ and $G_{a}$ can be analytically continued to the half-plane $\Re e s>0$. Note that $H(1)=A$, $G_{a}(1)=2^{-\varepsilon(a)} h(a)^{-1}$. By estimates of Selberg-Delange type (see [8], chap. II.5), $(2 \cdot 5)$ yields in turn

$$
\sum_{\substack{b \leqslant y \\(b, a)=1}} h\left(2^{\nu} b\right) \sim \frac{A y}{2^{\varepsilon(a)} h(a)} \quad(y \rightarrow \infty),
$$

and

$$
\sum_{\substack{b \leqslant x^{c} \\(a, b)=1,2 \mid a b}} \frac{h(b)}{b \log \left(x / a b^{2}\right)}=\frac{A}{4 h(a)} \log \left(\frac{1-v_{a}}{1-2 c-v_{a}}\right)+o(1) \quad(x \rightarrow \infty)
$$

and $v_{a}:=(\log a) / \log x$. Substituting this back into $(2 \cdot 4)$, we arrive at

$$
\begin{aligned}
S(x, c) & \leqslant\{2+o(1)\} x \sum_{a \leqslant x^{c}} \frac{1}{a \log (x / a)} \log \left(\frac{1-v_{a}}{1-2 c-v_{a}}\right) \\
& =\{2+o(1)\} x \int_{0}^{c} \log \left(\frac{1-v}{1-2 c-v}\right) \frac{\mathrm{d} v}{1-v} .
\end{aligned}
$$

We remark that with a little more care, the bound $1 / 5$ in the theorem may be replaced with $1 / 3$. 


\section{Further remarks}

In [3] it is shown that if $N$ is large, then for at least $0.0099 N$ values of $n \leqslant N$ we have $P(n)>P(n+1)$, and for at least $0.0099 N$ values of $n \leqslant N$ we have $P(n)<P(n+1)$. It follows from Theorem 1.2 that each inequality occurs on a set of integers $n$ of lower asymptotic density

$$
\log \left(\frac{1}{1-c}\right)-2 \int_{0}^{c} \log \left(\frac{1-v}{1-v-2 c}\right) \frac{\mathrm{d} v}{1-v}
$$

for each value of $c$ with $0<c<1 / 5$. The maximum of this expression is greater than 0.05544 so we have majorized the result from [3]. Presumably, the set $E$ of integers $n$ with $P(n)>P(n+1)$ has asymptotic density $1 / 2$. A general theorem of Hildebrand [4] also implies that $E$ has positive lower asymptotic density, but we did not check the numerical value that can be derived from this result.

In [3] it is shown that $P(n)<P(n+1)<P(n+2)$ holds infinitely often, and it was conjectured that so too $P(n)>P(n+1)>P(n+2)$ holds infinitely often. This conjecture was recently proved by Balog in [1].

We observe that the maximal value $A(N)$ corresponds to a sequence $\varepsilon=$ $\left\{\varepsilon_{n}\right\}_{1 \leqslant n<N}$ where $\varepsilon_{n} \in\{-1,1\}$.

Proposition 3.1. Let $N \geqslant 1$. There exists $\left\{\varepsilon_{n}\right\}_{1 \leqslant n<N} \in\{-1,1\}^{N-1}$ such that

$$
\frac{A(N)}{B(N)}=\prod_{1 \leqslant n<N}\left(\frac{n}{n+1}\right)^{\varepsilon_{n}} .
$$

Remark. Let $A_{0,1}(N)$ (respectively $A_{-1,1}(N), A_{-1,0}(N)$ ) the maximum of numerators where the exponents $\varepsilon_{n}$ are restricted to $\{0,1\}$ (respectively $\{-1,1\},\{-1,0\}$ ). By the proposition, we have $A_{-1,1}(N)=A(N)$ and

$$
\log A_{0,1}(N)=\frac{1}{2} \log A(N)+O(\log N)=\log A_{-1,0}(N)+O(\log N) .
$$

For example, if $\left\{\varepsilon_{n}\right\}_{1 \leqslant n<N} \in\{0,1\}^{N-1}$, we have $\left\{2 \varepsilon_{n}-1\right\}_{1 \leqslant n<N} \in\{-1,1\}^{N-1}$. Since the constant sequence -1 gives the numerator $N$, we deduce the result.

Proof. Take a sequence $\left\{\varepsilon_{n}\right\}_{1 \leqslant n<N} \in\{-1,0,1\}^{N-1}$ where some $\varepsilon_{n}=0$. Write the associated product as $A / B$ with $(A, B)=1$. If we let $\varepsilon_{n}=1$, the new numerator is

$$
\frac{A}{(A, n+1)} \times \frac{n}{(B, n)},
$$

while if we let $\varepsilon_{n}=-1$, the new numerator is

$$
\frac{A}{(A, n)} \times \frac{n+1}{(B, n+1)} .
$$


Assuming both of these expressions are smaller than $A$, we obtain

$$
n<(A, n+1)(B, n) \text { and } n+1<(A, n)(B, n+1) \text {. }
$$

Multiplying these inequalities and using $(A, B)=(n, n+1)=1$ we obtain

$$
n(n+1)<(A B, n(n+1)) \text {, }
$$

a contradiction. So we may choose $\varepsilon_{n} \in\{ \pm 1\}$ without decreasing the associated numerator. With this method we can replace each 0 value with \pm 1 and the value of the associated numerator will not decrease.

As pointed out in Section 2, the result of Theorem 1.2 can be improved by using more sophisticated sieve methods. Indeed, in the present treatment, we sift integers of the form $\left(a p_{1}+1\right) / b$ for fixed $a$ and $b$, and then sum over $a$ after having disposed of the $b$-sum by the Bombieri-Vinogradov theorem, thus applying this result to a sequence of length $x / a$ and hence of distribution level roughly $\sqrt{x / a}$. We could, alternatively, sift, for fixed $b$, the sequence comprising all $\left(a p_{1}+1\right) / b$ of a piece. We could then restrict the study to, say, $x / \log x<a p_{1} \leqslant x$. This corresponds to the Dirichlet convolution of two characteristic functions that are of Siegel-Walfisz type, and thus has distribution level $x^{1 / 2+o(1)}$ — see, e.g., [2] theorem 0(b). Such an approach yields, for any given $c_{0}, 0<c_{0}<\frac{1}{4}$, the upper bound

$$
S(x, c) \leqslant 2 x \log (1-2 c) \log (1-c)+o(x)
$$

uniformly for $0 \leqslant c \leqslant c_{0}$. In turn, this improvement allows the better value $K \approx 0.112945$ in Theorem 1.1, and ups the density in the first paragraph of this section to 0.05866. We warmly thank Étienne Fouvry for these observations.

\section{References}

[1] A. Balog, On triplets with descending largest prime factors, Studia Sci. Math. Hungar. 38 (2001), 45-50.

[2] E. Bombieri, J. B. Friedlander and H. Iwaniec, Primes in arithmetic progressions to large moduli, Acta Math. 156, n 3-4 (1986), 203-251.

[3] P. Erdös and C. Pomerance, On the largest prime factors of $n$ and $n+1$, Aequationes Math. 17 (1978), 311-321

[4] A. Hildebrand, On a conjecture of Balog, Proc. Amer. Math. Soc. $95, n^{\circ} 4$ (1985), $517-523$.

[5] H. Iwaniec, Rosser's sieve, Acta Arith. 36 (1980), 171-202.

[6] H. Iwaniec, A new form of the error term in the linear sieve, Acta Arith. 37 (1980), $307-320$.

[7] J.-L. Nicolas, Nombres hautement composés, Acta Arith. 49 (1988), 395-412.

[8] G. Tenenbaum, Introduction to analytic and probabilistic number theory, Cambridge studies in advanced mathematics, $n^{\circ} 46$, Cambridge University Press (1995).

Régis de la Bretèche

École Normale Supérieure

Mathématiques et Applications

45 , rue d'Ulm

75230 Paris Cedex 05, France

breteche@ens.fr
Carl Pomerance

Department of Mathematics

Dartmouth College

Hanover, NH 03755

USA

carlp@math.dartmouth.edu
Gérald Tenenbaum

Institut Élie Cartan

Université Henri Poincaré Nancy 1

BP 239

54506 Vandœuvre Cedex, France

gerald.tenenbaum@ciril.fr 